\title{
Autologous Stem Cell Application in Periodontal Regeneration Technique (SAI-PRT) Using PDLSCs Directly From an Extracted Tooth $\cdots$ An Insight
}

\author{
KL Vandana ${ }^{1}$, Rajendra Desai ${ }^{3}$, Priyanka Jairaj Dalvi ${ }^{2}$ \\ Department of ${ }^{1}$ Periodontics and ${ }^{2}$ Periodontics and ${ }^{3}$ Oral and Maxillofacial Surgery, College of Dental Sciences, Davangere
}

Periodontal regeneration represents the ultimate goal of periodontal therapy. The current regenerative techniques have limited success rates especially in advanced periodontal defects. Currently the research is focused on novel cell-based approaches for periodontal regeneration to overcome the limitations of existing treatment. The human clinical trial on stem cells based periodontal regeneration is promising. The plethora of animal studies provide sound evidence to support the belief that periodontal ligament stem cells (PDLSCs) can be used for periodontal regeneration. The direct application of autologous periodontal stem cells in treatment of intrabony defects is attempted for the first time in periodontal literature. Stem cell Application in Periodontal Regeneration Technique (SAI-PRT) using direct PDLSCs has overcome the limitations and concerns of ex- vivo stem cell culture methods like high cost, technique sensitivity, loss of stemness during cell passage, genetic manipulation and tumorigenic potential. Clinical feasibility, success and cost effectiveness over currently available techniques are encouraging. The clinical utility of this novel idea is recommended.

Keywords: Periodontal ligament progenitors, Regeneration, Periodontitis

\section{Introduction}

Periodontitis is an inflammatory disease and is the most common cause of tooth loss in adults. World Health Organization (WHO) has reported that $10 \sim 15 \%$ of the world populations suffer from severe periodontitis (1) moreover $50 \%$ of Indian population suffers from periodontitis (2). Periodontitis manifests clinically as loss of supporting structures including periodontal ligament and

\footnotetext{
Accepted for publication September 25, 2015, Published online November 30, 2015 Correspondence to $\mathrm{KL}$ Vandana

Department of Periodontics, College Of Dental Sciences, MCC 'B' Block, Bapuji Hospital rd., Karanataka 577004, Davangere Tel: + 91-08192-231285, 231029, Fax: +91-8192-236493

E-mail: vanrajs@gmail.com

(c) This is an open-access article distributed under the terms of the Creative Commons Attribution Non-Commercial License (http://creativecommons.org/ licenses/by-nc/4.0/), which permits unrestricted non-commercial use, distribution, and reproduction in any medium, provided the original work is properly cited.
}

alveolar bone. Following cause-related therapy, periodontal regeneration represents the ultimate goal of periodontal therapy which aims at re-formation of all components of the periodontium: gingival connective tissue, periodontal ligament, cementum and alveolar bone lost during the disease process.

\section{Periodontal tissue regeneration and Stem cell therapy}

Periodontal regeneration is especially challenging, as it requires predictable regeneration of three quite diverse and unique tissues (e.g., cementum, periodontal ligament, and bone) and a triphasic interface between these different tissues to guarantee the restoration of their complex structure $(3,4)$. The current regenerative techniques have limited success rates especially in advanced periodontal defects. In the view of this, current research is focused on novel cell-based approaches for periodontal regeneration 
to overcome the limitations of existing treatments.

Langer in 1993 proposed tissue engineering as a possible technique for regenerating lost periodontal tissues (5). This field consolidates materials science and biocompatibility, and integrates cells, natural or synthetic scaffolds, and specific signals to create new tissues. Tissue engineering represents one of the most sensational advances in regenerative medicine. However, there is scarcity of literature dealing application of tissue engineering for regeneration of periodontal tissues.

\section{Biomaterial assisted direct application of autologous periodontal ligament stem cells in intrabony defects}

The human periodontal ligament (PDL) encloses subpopulation of stem cells which responsible for maintaining and regenerating periodontal tissue structure and function. These cells exhibit multipotency, as demonstrated by their ability to differentiate into osteoblasts, fibroblasts and tooth cementoblasts and to form cementum- and PDL-like tissues, and are generally termed periodontal ligament stem cells (PDLSCs.) They were first isolated from the PDL tissue of extracted human third molar teeth (6). In addition to the periodontal ligament of the root surface following tooth extraction, the PDL remaining on the alveolar bone surface of the extraction sockets has also been used to isolate PDLSCs (7).

The human clinical trial on stem cells based periodontal regeneration is promising. The plethora of animal studies provide sound evidence to support the belief that PDLSCs can be used for periodontal regeneration (8). The implantation of cells into the periodontal defect is done via either biomaterial-free or biomaterial-based approaches (9) also these host derived cells can either be subjected isolation, ex-vivo expansion (stem cell culture) and re-implantation of these cells into periodontal wound /defect or injected directly as a suspension or delivered by biomaterial scaffolds or cell carriers (10).

Primary cultures using ex vivo methods PDLSCs yielded small cell numbers (an average of 1,250 cells) (11) which is less periodontal regeneration (at least $4 \times 10^{6}$ cells). Thus, PDLSCs must proliferate at least 12 population doublings before application (12).

Although the ex vivo expansion of stem/ progenitor cells is necessary, these cells typically reduce their ability to self-renew and proliferate during passaging. The major difficulties facing widespread clinical implementation of existing cell delivery strategies for periodontal therapy are the qualification, cost and time required for ex vivo cul- ture of a patient's autologous cells prior to re-implantation, and the potential risks and availability constraints associated with transplanting allogeneic (foreign) cells (10). In addition to reduced washing out of the implanted cells in an ex vivo cultures, the use of a carrier material enables the introduction of additional biological cues such as the release of angiogenic factors to improve connection to the host vascular network or factors that drive a differentiation process $(13,14)$. Hence a gelatine sponge owing to its flexibility, biocompatibility, and biodegradability, and potential to be used as a scaffold to support osteoblasts and to promote bone regeneration in defective areas was considered (15).

The human clinical trial on stem cells based periodontal regeneration is promising. In the first experiment in humans using autologous periodontal ligament derived -derived cells, including PDLSCs, to reconstruct the periodontal intra-bony defects in three patients, the periodontal ligament stem cells were cultured (ex-vivo) and then transplanted which induced a significant improvement of periodontal disease, suggesting that PDLSCs transplantation may be an efficacious and safe alternative for the treatment of human periodontitis (12).

Periodontal regeneration is defined histologically as regeneration of the tooth's supporting tissues, including alveolar bone, periodontal ligament, and cementum over a previously diseased root surface (16). PDLSCs are multipotent and have demonstrated their ability to differentiate into osteoblasts, fibroblasts and tooth cementoblasts and to form cementum- and periodontal ligament (PDL)-like tissues (6). Cementum appear to play a critical role in the regeneration of the tooth attachment apparatus. Cementum is the site where soft-tissue attachment has to be re-established, and cementum matrix is a rich source of many growth factors such as IGF, FGF, BMPs and many more which influence the activities of various periodontal cell types $(17,18)$.

Based on current literature on use of ex vivo culture and associated problems, a humble attempt was made to harvest autologous PDLSCs for direct application using Abgel ${ }^{\text {RCTM }}$ (gelatin sponge-Shri Gopal Krishna Labs Pvt. Ltd. Mumbai India.) as scaffold in regeneration of intrabony periodontal defect bypassing ex vivo culture. We used periodontal ligament of an extracted impacted wisdom tooth to restore the periodontal defect of another molar of the same patient. The trial was based on tissue engineering triad using PDLSCs from impacted third molar (cells), Abgel ${ }^{\text {RCTM }}$ (gelatine sponge - scaffold) and cementum scrapings (which consist of variety of signaling molecules e.g. IGF, VEGF etc. resulted in successful clinical and radiographic pa- 
rameters such as clinical attachment gain, decreased probing pocket depth and satisfactory defect fill of intrabony defect when evaluated for a period 1 year. (unpublished data). The direct application of autologous periodontal stem cells in treatment of intrabony defects was attempted for the first time in periodontal literature.

The acceleration of a patient's endogenous regenerative mechanisms by recruiting host stem/ progenitor cells, for periodontal regeneration has been considered. Although direct application of periodontal ligament stem cell therapy looks promising but it is still in its stage of infancy, more work is needed in this area to validate the results. Stem cell Application in Periodontal Regeneration Technique (SAI-PRT) using direct PDLSCs has overcome the limitations and concerns of ex- vivo stem cell culture methods like high cost, technique sensitivity, loss of stemness during cell passage, genetic manipulation and tumorigenic potential (19).

\section{Conclusion}

Although in vitro and pre-clinical animal studies have been pursued with a large and diverse collection of scaffolds, cells and biomolecules, and clinical studies are beginning to emerge, the field of periodontal tissue engineering and regeneration is fragmented to the point that a clear translational roadmap has yet to emerge.

Innovative stem cell therapy by great researchers has been a boon and an interesting development in various treatment modalities. A simple task of PDLSCs procurement and immediate placement are the major advantages of the current concept autologous Stem cell Application in Periodontal Regeneration Technique (SAI-PRT) has emerged a constructive avenue in treatment of periodontal osseous defects. Moreover the clinical feasibility, success and cost effectiveness over currently available techniques are encouraging. The clinical utility of this novel idea is recommended.

\section{References}

1. Petersen PE, Ogawa H. Strengthening the prevention of periodontal disease: the WHO approach. J Periodontol 2005; 76:2187-2193

2. Agarwal V, Khatri M, Singh G, Gupta G, Marya CM, Kumar V. Prevalence of Periodontal Diseases in India. J Oral Health Comm Dent 2010;4 Suppl:7-16

3. Young CS, Abukawa H, Asrican R, Ravens M, Troulis MJ,
Kaban LB, Vacanti JP, Yelick PC. Tissue-engineered hybrid tooth and bone. Tissue Eng 2005;11:1599-1610

4. Chen FM, Zhang J, Zhang M, An Y, Chen F, Wu ZF. A review on endogenous regenerative technology in periodontal regenerative medicine. Biomaterials 2010;31:7892-7927

5. Nakahara T. A review of new developments in tissue engineering therapy for periodontitis. Dent Clin North Am 2006;50:265-276

6. Seo BM, Miura M, Gronthos S, Bartold PM, Batouli S, Brahim J, Young M, Robey PG, Wang CY, Shi S. Investigation of multipotent postnatal stem cells from human periodontal ligament. Lancet 2004;364:149-155

7. Wang L, Shen H, Zheng W, Tang L, Yang Z, Gao Y, Yang Q, Wang C, Duan Y, Jin Y. Characterization of stem cells from alveolar periodontal ligament. Tissue Eng Part A 2011;17:1015-1026

8. Yan XZ, Yang F, Jansen JA, de Vries Rob BM, van den Beucken Jeroen JJP. Cell-based approaches in periodontal regeneration: a systematic review and meta-analysis of periodontal defect models in animal experimental work. Tissue Eng Part B Review 2015;21:411-426.

9. Lin NH, Gronthos S, Bartold PM. Stem cells and future periodontal regeneration. Periodontol 2000 2009;51:239-251

10. Chen FM, Sun HH, Lu H, Yu Q. Stem cell-delivery therapeutics for periodontal tissue regeneration. Biomaterials 2012;33:6320-6344

11. Iwata T, Yamato $M$, Zhang Z, Mukobata S, Washio K, Ando T, Feijen J, Okano T, Ishikawa I. Validation of human periodontal ligament-derived cells as a reliable source for cytotherapeutic use. J Clin Periodontol 2010;37:1088-1099

12. Feng F, Akiyama K, Liu Y, Yamaza T, Wang TM, Chen JH, Wang BB, Huang GT, Wang S, Shi S. Utility of PDL progenitors for in vivo tissue regeneration: a report of 3 cases. Oral Dis 2010;16:20-28

13. Chen FM, Zhang M, Wu ZF. Toward delivery of multiple growth factors in tissue engineering. Biomaterials 2010;31: 6279-6308

14. Soto-Gutierrez A, Yagi H, Uygun BE, Navarro-Alvarez N, Uygun K, Kobayashi N, Yang YG, Yarmush ML. Cell delivery: from cell transplantation to organ engineering. Cell Transplant 2010;19:655-665

15. Rohanizadeh R, Swain MV, Mason RS. Gelatin sponges (Gelfoam) as a scaffold for osteoblasts. J Mater Sci Mater Med 2008;19:1173-1182

16. Consensus report. Periodontal regeneration around natural teeth. Ann Periodontol 1996;1:667-670

17. Narayanan AS, Bartold PM. Biochemistry of periodontal connective tissues and their regeneration: a current perspective. Connect Tissue Res 1996;34:191-201

18. Saygin NE, Giannobile WV, Somerman MJ. Molecular and cell biology of cementum. Periodontol 2000 2000;24:73-98

19. Zhu W, Liang M. Periodontal ligament stem cells: current status, concerns, and future prospects. Stem Cells Int 2015; 2015:972313 